\title{
Prophylactic Antiplatelet Medication in Endovascular Treatment of Intracranial Aneurysms: Low-Dose Prasugrel versus Clopidogrel
}

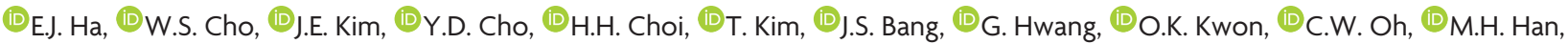 \\ and 1 H.S. Kang
}

\begin{abstract}
BACKGROUND AND PURPOSE: Prophylactic antiplatelet medication is beneficial in decreasing thromboembolic complications during endovascular treatment of unruptured intracranial aneurysms. The efficacy may be limited by variability of individual response to antiplatelet medication, especially clopidogrel. We compared the efficacy of 2 antiplatelet medications, low-dose prasugrel and clopidogrel, in patients undergoing endovascular treatment of unruptured aneurysms.
\end{abstract}

MATERIALS AND METHODS: From November 2014 to July 2015, 194 patients with a total of 222 unruptured aneurysms underwent endovascular treatment at a single institution. Laboratory and clinical data from the prospectively maintained registry were used in this study. Antiplatelet medication was given the day before endovascular treatment (prasugrel $20 \mathrm{mg}$ or $30 \mathrm{mg}$ or clopidogrel $300 \mathrm{mg}$ ). Response to the antiplatelet medication was measured by the VerifyNow system. Periprocedural adverse event rates between the 2 groups were compared.

RESULTS: There were no significant differences in the baseline characteristics of patients and aneurysms between the 2 groups. The P2Y12 reaction unit values were lower (clopidogrel group versus prasugrel group, $242.7 \pm 69.8$ vs $125.7 \pm 79.4 ; P<.0001$ ) and percentage inhibition values were higher $(22.1 \% \pm 19.7 \%$ vs $60.2 \pm 24.7 \% ; P<.0001)$ in the prasugrel group. There were no thromboembolic events, but there was 1 procedural bleed in each group, without any clinical consequences.

CONCLUSIONS: The prasugrel group showed more effective and consistent platelet inhibition. We may omit the antiplatelet response assay with the low-dose prasugrel premedication before the endovascular treatment of patients with unruptured aneurysms. Further study is required to determine whether there is benefit of this strategy regarding clinical outcome.

ABBREVIATIONS: CPG = clopidogrel; PRU $=$ P2Y12 reaction unit; PSG $=$ prasugrel

C oil embolization is accepted as a safe and effective treatment in patients with intracranial aneurysms. ${ }^{1}$ However, it is not without risk, and thromboembolism during the procedure is a serious and common complication of coil embolization. $^{2}$ Increasing evidence has demonstrated the efficacy of prophylactic antiplatelet therapy in patients undergoing endovascular treatment of unruptured intracranial aneurysms with or without stent assistance. ${ }^{3-7}$ One of the representative antiplatelet agents is clopidogrel (CPG), which inhibits the P2Y12

Received September 15, 2015; accepted after revision May 9, 2016.

From the Departments of Neurosurgery (E.J.H., W.S.C., J.E.K., H.S.K.) and Radiology (Y.D.C., M.H.H.), Seoul National University College of Medicine, Seoul National University Hospital, Seoul, Korea; Department of Neurosurgery (H.H.C.), Dongguk University Ilsan Hospital, Gyeonggi, Korea; and Department of Neurosurgery (T.K., J.S.B., G.H., O.K.K., C.W.O.), Seoul National University Bundang Hospital, Gyeonggi, Korea.

Please address correspondence to Hyun-Seung Kang, MD, PhD, Department of Neurosurgery, Seoul National University College of Medicine, Seoul National University Hospital, 101 Daehak-ro, Jongno-gu, Seoul 03080, Korea; e-mail: hsk4428@yahoo.com

http://dx.doi.org/10.3174/ajnr.A4864 adenosine diphosphate receptor on the platelet cell membrane. CPG has become a standard medication for patients with risk of cerebral infarction and myocardial infarction. ${ }^{8,9}$ To minimize the thromboembolic complications during the procedure, an antiplatelet protocol centered upon CPG has been adopted. $^{5,6,10}$

However, the efficacy of prophylactic CPG therapy is variable among patients. Some patients show resistance to CPG, and procedure-related thromboembolic events occur more frequently among them. ${ }^{10-13}$ Some reports have demonstrated higher numbers of acute ischemic lesions in patients with $\mathrm{CPG}$ resistance. ${ }^{14,15}$

Recently, a new generation of P2Y12 adenosine diphosphatereceptor antagonists has been developed. One such drug is prasugrel (PSG). ${ }^{16}$ The purpose of this study was to identify the role of low-dose PSG premedication in preventing thromboembolic events and to compare its effectiveness with CPG. We performed a comparative study between a low-dose PSG group and a CPG group in terms of platelet-response variability and periprocedural adverse event rates. 


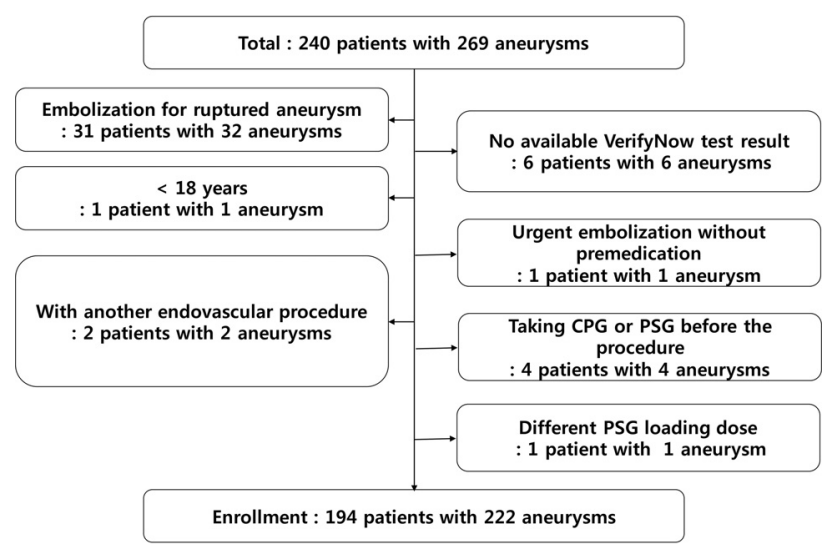

FIG 1. Patient selection flowchart.

\section{MATERIALS AND METHODS}

Study Design and Patient Population

After approval by the Seoul National University Hospital Institutional Review Board (No. 1503-090-657), we retrospectively identified 240 consecutive patients (with 269 aneurysms) who underwent endovascular treatment between November 2014 and July 2015 according to a prospectively maintained data base. The study included patients who were older than 18 years and were treated for unruptured intracranial aneurysms. Excluded were those who had ruptured, infectious, or traumatic aneurysms; who had a previous history of intracranial hemorrhage; who underwent parent arterial occlusion; who underwent urgent treatment without antiplatelet premedication; who had no platelet function test results available; who took a different PSG loading dose (15 $\mathrm{mg}$ ); and who had already been taking CPG or PSG for other reasons before the aneurysmal treatment. As a result, 194 patients with a total of 222 aneurysms were enrolled (Fig 1).

The patients were divided into the CPG group and the lowdose PSG group; the choice of antiplatelet agents was determined by the referring physicians. The CPG group included 96 patients (with 106 aneurysms) who received CPG as a premedication, and the low-dose PSG group included 98 patients (with 116 aneurysms).

\section{Periprocedural Antiplatelet Medication}

In the CPG group, a 300-mg loading dose of CPG was given the day before the procedure, and an additional $75 \mathrm{mg}$ of CPG was given on the morning of the procedure. If the patient showed a high $\mathrm{P} 2 \mathrm{Y} 12$ reaction unit (PRU) value, we gave an additional antiplatelet agent (aspirin). If a stent-assisted procedure was anticipated, we routinely gave additional aspirin to the patient without considering the PRU values, and if the patient was a poor responder to CPG (ie, PRU > 285), we added cilostazol (200 mg per day). ${ }^{10}$ Conversely, in the PSG group, the patients received typically a 20-mg loading dose of PSG the day before the procedure and an additional $5 \mathrm{mg}$ of PSG was administered on the morning of the procedure.

After the embolization procedures, antiplatelet medication was continued if necessary, depending on the presence of underlying atherosclerotic steno-occlusive diseases, stent implantation, or coil loop protrusion during the procedure. Patients in the CPG group undergoing stent implantation were recommended dual- or triple-antiplatelet (for CPG poor responders) agents for 3 months. Patients in the PSG group undergoing stent implantation received $5 \mathrm{mg}$ of PSG for 3 months; then, life-long aspirin medication was recommended.

\section{Blood Sample Acquisition and Platelet Activity Measurement}

Whole blood was obtained 6 hours after antiplatelet agent loading in both groups. Residual platelet reactivity was measured by using the VerifyNow assay (Accumetrics, San Diego, California). We obtained 3 values: platelet reactivity to isothrombin receptor-activating peptide, or BASE; PRU; and percentage inhibition. BASE is a platelet reaction unit and serves as an estimate of baseline platelet reactivity. PRU reflects residual P2Y12 receptor activity. Percentage inhibition is a calculated value from a formula:

Percentage Inhibition $=[($ BASE - PRU $) /$ BASE $] \times 100$

Therefore, high PRU and low percentage inhibition indicate a lower effectiveness of adenosine-diphosphate receptor-antagonist therapy.

\section{Coil Embolization Procedure and Procedure-Related Complications}

Endovascular coil embolization was performed by using a standardized protocol in a neuroangiography suite. Most procedures were performed on patients under general anesthesia. Systemic anticoagulation with heparin was done from the start of the procedure. Heparin was usually administered as a bolus of $3000 \mathrm{IU}$ intravenously after insertion of the introducer sheath and infused additionally at a rate of 1000 IU per hour with monitoring of the activated clotting time. The target activated clotting time was 250-300 seconds.

A simple coiling procedure (single microcatheter placement and coil deployment), multiple microcatheter technique, balloon-assisted technique, stent-assisted coil embolization, combination of the former techniques, and flow diversion were used at the discretion of the treating physician.

The procedure-related thromboembolism was defined as thrombus formation and/or distal embolism observed during the procedure or clinically recognized ischemic deficits (including transient ischemic attack) that occurred within 60 days of the procedure. Procedural thromboembolism included procedural clot or any ischemic events found after treatment, and periprocedural thromboembolism included the vascular territory-specific events related to the treated lesions within 60 days of the procedure. In the latter, alternative etiologies were ruled out after evaluation by stroke neurologists. Events of procedure-related bleeding also were recorded.

\section{Statistical Analysis}

Categoric data were presented as frequencies and group percentages, and continuous data were presented as mean $\pm \mathrm{SD}$. The Fisher exact test or $\chi$-square test was used for comparison of discrete data. An independent $t$ test was conducted to compare the unpaired continuous data. All tests were 2-tailed, and statistical significance was considered at $P<.05$. Analyses were performed by using Statistical Package for the Social Sciences for Windows version 20.0 (IBM, Armonk, New York). Bleeding events and ad- 
Table 1: Baseline characteristics of patients ${ }^{a}$

\begin{tabular}{lccc}
\hline \multicolumn{1}{c}{ Characteristic } & CPG & PSG & P Value \\
\hline No. of patients & 96 & 98 & \\
No. of aneurysms & 106 & 116 & \\
Sex (F/M) & $63 / 33$ & $68 / 30$ & .646 \\
Age (yr) & $56.4 \pm 11.4$ & $57.7 \pm 10.5$ & .418 \\
Body weight $(\mathrm{kg})$ & $62.3 \pm 9.7$ & $61.8 \pm 10.8$ & .765 \\
Body mass index & $24.5 \pm 3.1$ & $24.2 \pm 4.0$ & .573 \\
Hypertension & $38.5 \%$ & $43.9 \%$ & .469 \\
Diabetes & $6.3 \%$ & $8.2 \%$ & .783 \\
Hyperlipidemia & $53.1 \%$ & $50.0 \%$ & .670 \\
Smoking & $31.3 \%$ & $26.5 \%$ & .631 \\
Triglycerides $(\mathrm{mg} / \mathrm{dL})$ & $106.9 \pm 52.1$ & $117.3 \pm 67.5$ & .234 \\
HDL cholesterol $(\mathrm{mg} / \mathrm{dL})$ & $51.1 \pm 13.7$ & $56.0 \pm 33.9$ & .190 \\
LDL cholesterol (mg/dL) & $99.1 \pm 29.6$ & $107.5 \pm 29.3$ & .052 \\
\hline
\end{tabular}

Note:- HDL indicates high-density lipoprotein; LDL, low-density lipoprotein.

${ }^{a}$ All the continuous variables are presented as mean $\pm S D$.

Table 2: Baseline characteristics of aneurysms

\begin{tabular}{lccc}
\hline \multicolumn{1}{c}{ Characteristic } & CPG & PSG & P Value \\
\hline Aneurysm volume $\left(\mathrm{mm}^{3}\right)$ & $0.121 \pm 0.402$ & $0.141 \pm 0.674$ & .807 \\
Location (no. [\%]) & & & .867 \\
Internal carotid artery & $54(50.9)$ & $56(48.3)$ & \\
Anterior cerebral artery & $29(27.4)$ & $30(25.9)$ & \\
Middle cerebral artery & $13(12.3)$ & $19(16.4)$ & \\
Posterior circulation & $10(9.4)$ & $11(9.5)$ & \\
Previously ruptured & 3 & 5 & .555 \\
Shape & 103 & 112 & .686 \\
Saccular & 1 & 0 & \\
Fusiform & 1 & 1 & \\
Dissecting & 1 & 2 & \\
Post-clip remnant & 0 & 1 & \\
Partially thrombosed & &
\end{tabular}

Table 3: Procedural characteristics in each group

\begin{tabular}{lrrc}
\hline \multicolumn{1}{c}{ Characteristic } & CPG & PSG & P Value \\
\hline Treatment times & & & .270 \\
First treatment & 98 & 101 & \\
Repeated treatment & 8 & 15 & \\
Treatment modality & & & .116 \\
Simple coiling & 40 & 46 & \\
Stent-assisted & 23 & 19 & \\
Multiple microcatheter & 23 & 31 & \\
Balloon-assisted & 8 & 16 & \\
Combined $^{\text {a }}$ & 10 & 4 & \\
Flow diversion & 2 & 0 & \\
\hline
\end{tabular}

${ }^{a}$ Combined procedures are stent-assisted and balloon-assisted, multiple microcatheter and balloon-assisted, or multiple microcatheter and stent-assisted.

verse thromboembolic events were reported in a descriptive manner.

\section{Approval}

This study was conducted according to the principles outlined in the Declaration of Helsinki. The protocol was approved by Seoul National University Hospital's Institutional Review Board (No. 1503-090-657).

\section{RESULTS}

The baseline characteristics of patients, treated aneurysms, and procedural characteristics are summarized in Table 1, Table 2, and Table 3, respectively. No significant differences were found in the baseline characteristics between the PSG and CPG groups. In the PSG group, 90 patients received 20-mg loading doses and 8
Table 4: Comparison of procedure-related complications

\begin{tabular}{lccc}
\hline \multicolumn{1}{c}{ Complication } & CPG & PSG & P Value \\
\hline Thromboembolism & 0 & 0 & NS \\
Aneurysm perforation & $1(0.9 \%)$ & $1(0.9 \%)$ & NS \\
\hline
\end{tabular}

Note:-NS indicates not significant.

Table 5: Comparison of platelet function test results using VerifyNow P2Y12 assay

\begin{tabular}{lccc}
\multicolumn{1}{c}{ Test } & CPG & PSG & P Value \\
\hline BASE & $311.8 \pm 41.1$ & $313.7 \pm 41.6$ & .753 \\
PRU & $242.7 \pm 69.8$ & $125.7 \pm 79.4$ & $<.001$ \\
Percentage inhibition (\%) & $22.1 \pm 19.7$ & $60.2 \pm 24.7$ & $<.001$ \\
\hline
\end{tabular}

Note:-BASE indicates platelet reactivity to isothrombin receptor-activating peptide.

patients received 30-mg loading doses. There were 32 stent-assisted procedures performed in the CPG group and 23 performed in the PSG group.

There were no procedure-related thromboembolic events (Table 4). There was 1 procedural bleeding event in each group; in the CPG group, it was an aneurysmal rupture during the procedure. Diffuse SAH was identified on immediate postoperative CT scan. In the PSG group, there was also 1 hemorrhagic complication case. A small amount of SAH developed because of microguidewire perforation of the aneurysmal sac during the procedure. However, they were not clinically significant. There was no procedure-related permanent morbidity or mortality in either group.

The platelet function test results are summarized in Table 5. There were no significant differences in the BASE values (CPG group vs PSG group, $311.8 \pm 41.1$ vs $313.7 \pm 41.6$, respectively; $P=.753)$, but PRU values were significantly lower $(242.7 \pm 69.8$ vs $125.7 \pm 79.4$, respectively; $P<.0001)$ and percentage inhibition values were higher $(22.1 \% \pm 19.7 \%$ vs $60.2 \% \pm 24.7 \%$, respectively; $P<.0001)$ in the PSG group. The histogram of the PRU values of the CPG group showed a bell-shaped distribution (Fig 2). The PSG group showed a distribution of PRU values shifted to the left side compared with the CPG group. When we set a PRU cutoff value of 285 to determine the resistance to adenosine-diphosphate antagonists, ${ }^{10} 30.2 \%$ (29 of 96) and 1.0\% (1 of 98) of the patients in the CPG group and the PSG group, respectively, showed resistance $(P=.001)$.

In the CPG group, 54 patients $(56 \%)$ were given dual-antiplatelet agents, and 11 patients (12\%) were given triple-antiplatelet agents. The remaining 31 patients (32\%) were given CPG only. In the PSG group, 89 of 98 patients (91\%) were given PSG only; only 9 patients had dual-antiplatelet agents. The rate of using multiple antiplatelet agents was significantly higher in the CPG group $(P<.001)$.

When we analyzed the antiplatelet effect of PSG according to the loading doses (30 mg versus $20 \mathrm{mg}$ ), there were meaningful differences in PRU values between the 2 groups. The PRU value was significantly lower in the $30-\mathrm{mg}$ group ( $30 \mathrm{mg}$ versus $20 \mathrm{mg}$, $68.7 \pm 72.3$ vs $132.2 \pm 80.7 ; P=.0046)$. We also analyzed the relationship between clinical and laboratory characteristics and PRU in the PSG group (Table 6). PRU values were significantly lower in those with lower body weight $(<60 \mathrm{~kg} ; P<.001)$, statin users $(P=.02)$, smokers $(P<.001)$, and those with higher low density-lipoprotein levels $(P<.001)$. 


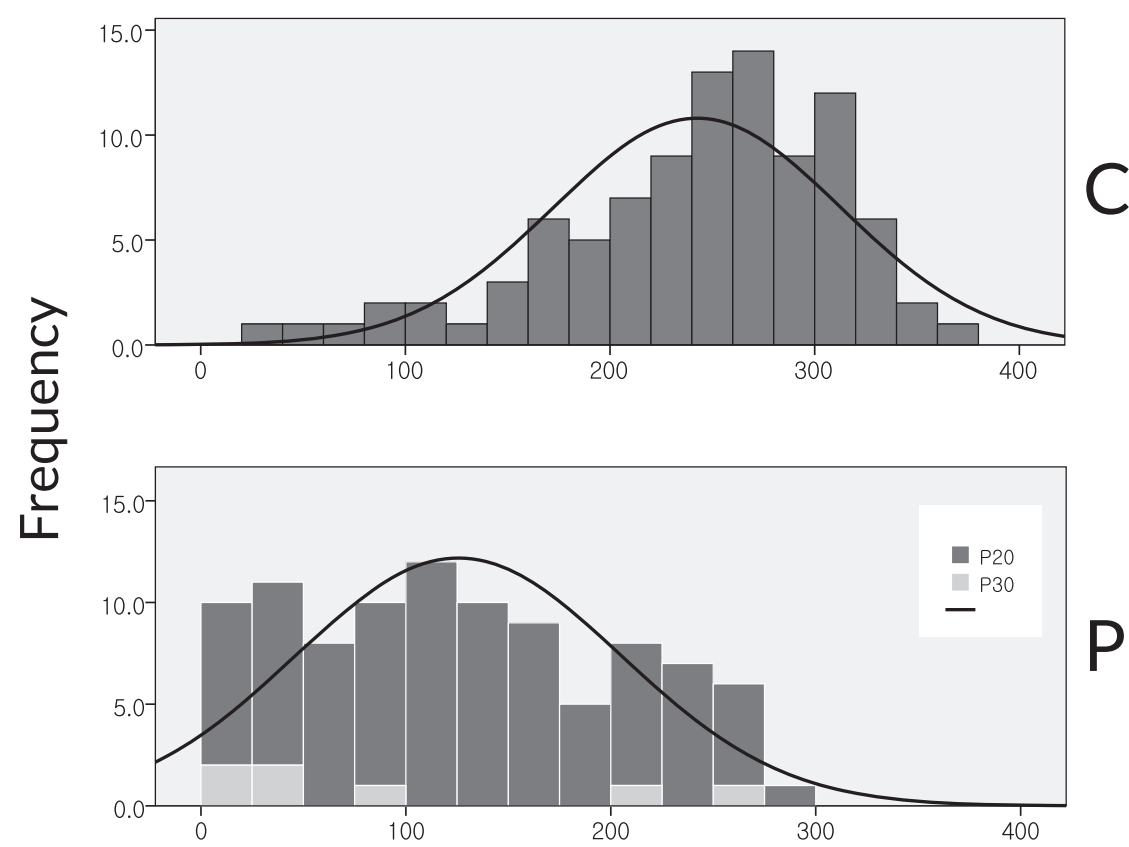

FIG 2. PRU value distribution in CPG and PSG groups. $C$ indicates CPG group; P, PSG group; P20, PSG 20-mg loading dose; P30, PSG 30-mg loading dose.

Table 6: Relations between patient characteristics and PRU in the PSG group

\begin{tabular}{|c|c|c|}
\hline Clinical and Laboratory Variables $^{a}$ & $P$ Value & PRU \\
\hline $\operatorname{Sex}(M)$ & .18 & $129.6 \pm 49.0$ vs $130.3 \pm 68.0$ \\
\hline Age $(<65 \mathrm{yr})$ & .17 & $122.6 \pm 59.4$ vs $148.0 \pm 65.4$ \\
\hline Body weight (<60 kg) & $<.001$ & $102.3 \pm 54.5$ vs $156.7 \pm 55.2$ \\
\hline Hypertension (no) & .20 & $120.6 \pm 62.2$ vs $142.0 \pm 60.1$ \\
\hline Diabetes mellitus (yes) & .31 & $127.5 \pm 63.8$ vs $157.0 \pm 37.6$ \\
\hline Statin use (yes) & .02 & $109.5 \pm 42.8$ vs $148.2 \pm 72.3$ \\
\hline Smoking status (current or past smoker) & $<.001$ & $77.4 \pm 26.1$ vs $148.2 \pm 61.5$ \\
\hline Alcohol intake (yes) & .24 & $110.6 \pm 106.5$ vs $135.0 \pm 50.6$ \\
\hline Hematocrit level (<38\%) & .84 & $128.5 \pm 69.4$ vs $131.8 \pm 55.0$ \\
\hline Platelet count $\left(<227 \times 10^{3}\right)$ & .58 & $125.6 \pm 61.5$ vs $135.0 \pm 63.0$ \\
\hline HDL cholesterol level ( $<40$ mg/dL) & .89 & $125.6 \pm 50.1$ vs $129.2 \pm 64.2$ \\
\hline LDL cholesterol level ( $\geq 130$ mg/dL) & $<.001$ & $69.5 \pm 21.1$ vs $145.9 \pm 59.1$ \\
\hline
\end{tabular}

Note:-HDL indicates high-density lipoprotein; LDL, low-density lipoprotein.

${ }^{a}$ A group of patients denoted within the parentheses showed decreased residual platelet activity.

\section{DISCUSSION}

\section{Antiplatelet Resistance and Coil Embolization of Unruptured Aneurysms}

In previous studies, CPG premedication contributed to reduction in procedure-related thromboembolism without increasing the risk of procedural bleed. ${ }^{4-6}$ In a study by Kang et al, ${ }^{6}$ the rates of absolute and relative risk reduction were 5.2\% (from $12.6 \%$ to $7.4 \%$ ) and $41 \%$, respectively. Furthermore, the logistic regression analysis proved CPG premedication as an independent factor related to thromboembolic events. Thus, the classic protocol for antiplatelet prophylaxis in the neurointerventional field has mainly been composed of CPG.

However, there was a problem of variable responsiveness to CPG. Though there are controversies on the utility of plateletinhibition testing, variable responsiveness to CPG has led practitioners to perform laboratory tests to evaluate platelet reactivity after CPG administration, especially before interventional procedures. Several studies in the field of cardiology demonstrated that high PRU values were associated with increased periprocedural myocardial infarction as well as mortality after percutaneous coronary intervention. ${ }^{17-19}$ In the neurointerventional field, a series of studies since the first publication in this field in 2010 have demonstrated the association between antiplatelet drug resistance and periprocedural ischemic events in reference to endovascular treatment of intracranial aneurysms. ${ }^{10,13-15,19-21}$ Kang et $\mathrm{al}^{10}$ showed that procedure-related thromboembolic events occurred more frequently in patients with higher PRU, especially in the fourth quartile. Furthermore, in a randomized clinical trial, modification of antiplatelet treatment based on the functional assay provided benefit in terms of thromboembolic event rate. ${ }^{22}$ Additional doses or other kinds of antiplatelet agents would bring complexities of the premedication protocols and increased costs, which prompted us to search for an effective antiplatelet regimen with less variability.

\section{Antiplatelet Premedication with Low-Dose PSG}

PSG, a third-generation thienopyridine, has shown significant reduction in rates of ischemic events in patients undergoing coronary intervention. ${ }^{23-25}$ It also has been shown to increase the bleeding risk in patients with acute coronary syndrome. $^{25}$ Both CPG and PSG are prodrugs that require biotransformation to active metabolites by cytochrome P450 enzymes. ${ }^{16}$ Though the active metabolites of both drugs have a similar affinity for the $\mathrm{P} 2 \mathrm{Y} 12$ receptor in vitro, the in vivo difference in response appears to be mediated by differences in the metabolic pathways leading to the formation of the active metabolites. Hepatic esterases shunt most (approximately $85 \%$ ) of CPG to a dead-end inactive pathway, with the remaining prodrug requiring 2 separate cytochrome $\mathrm{P} 450$-dependent oxidative steps. ${ }^{26}$ In contrast, esterases are part of the activation pathway with PSG, and PSG is oxidized to its active metabolite in a single cytochrome P450-dependent step without an apparent dead-end inactive pathway. ${ }^{27}$ The genes that encode the cytochrome P450 enzymes are polymorphic, with certain alleles demonstrated to confer reduced enzymatic function, thereby interfering with the production of the drug metabolites. The action of CPG is more dependent on the genetic polymorphism of the cytochrome P450 enzyme, and it varies among people.

The occasional poor response to CPG gives us concern for the increased risk of procedural complications, and the complicated individual tailoring sometimes confuses medical personnel in our daily practice. PSG has demonstrated a superior antiplatelet effect 
compared with CPG, especially in cardiology. However, usage of PSG in the field of cerebrovascular diseases is quite limited for fear of the risk of intracranial hemorrhage. ${ }^{25}$

In the current study, we focused on the role of low-dose PSG premedication in patients undergoing coil embolization for unruptured intracranial aneurysms. This study is the largest among the clinical studies on the usage of prophylactic PSG in the neurointerventional field ${ }^{28,29}$ and shows the efficacy and safety of the reduced-dose PSG regimen, composed of a 20-mg loading dose and a 5-mg maintenance dose. (In the TRITON-TIMI 38 trial, the medication protocol was a 60 -mg loading dose and a $10-\mathrm{mg}$ daily maintenance dose. ${ }^{25}$ ) In our series, we used $30 \mathrm{mg}$ as the loading dose during the early phase. After a few cases, we found that PRU values were sometimes too low. All 7 patients taking the $30-\mathrm{mg}$ loading dose showed a "hyper-response" (PRU value $<240)^{10}$ and 4 showed PRU values $<60$ (a criterion for "hyper-response" in another publication). ${ }^{12}$ PRU values were below 10 in 2 patients and between 10 and 60 in another 2 . Therefore, we reduced the loading dose of PSG to $20 \mathrm{mg}$. The mean PRU values were $68.7 \pm$ 72.3 and $132.2 \pm 80.7$ in the $30-\mathrm{mg}$ and 20 -mg loading groups, respectively $(P=.0046)$. We could infer that the optimal loading dose of PSG for sufficient suppression of platelet function without increased bleeding risk in our cohort would be less than $30 \mathrm{mg}$.

In the current study, patients in the PSG group consistently showed significantly lower PRU values. The overall distribution of PRU values was shifted to the left, as shown in Fig 2. On the basis of VerifyNow test results, only 1 patient showed high PRU over the cutoff value of 285 (the patient's PRU was 295 and percentage inhibition was 15\%).

Most patients in the CPG group (68\%) were given additional (1 or 2) antiplatelet agents. Conversely, only 9 patients in the PSG group (9\%) took dual-antiplatelet agents, and 7 of them had already taken aspirin for other medical reasons. The single-agent therapy with low-dose PSG showed at least comparable protective antithromboembolic effects compared with CPG-based combination antiplatelet therapy. In terms of side effects of PSG, especially procedural-related bleeding, there was only 1 event, which did not lead to any clinical impairment of the patient.

CPG-based combination antiplatelet therapy is a widely used prophylactic regimen for stent-assisted coil embolization procedures. In our series, 14 patients underwent stent-supported coil embolization under low-dose PSG premedication only. They did not show any thromboembolic complications during the procedure and the following 2 months. Single-antiplatelet premedication with PSG seems to be sufficient even in cases requiring stentsupported coil embolization. Though PSG is more expensive than $\mathrm{CPG}$, the total medication cost would be similar considering the combination antiplatelet therapy in the CPG group. The antiplatelet medication protocol will become simpler with use of the "low-dose PSG protocol." In addition, we also might reduce cost by omitting the platelet function assay because consistent platelet inhibition was verified with PSG.

The limitations of the current study include its retrospective nature and relatively small number of patients. Further study is anticipated to see whether the low-dose PSG protocol can bring benefits regarding the clinical outcome, especially in the setting of a larger, prospective, randomized, multicenter trial.

\section{CONCLUSIONS}

In our study, the PSG group showed more effective and consistent platelet inhibition than the CPG group. With this antiplatelet regimen, we may omit the antiplatelet response assay and additional antiplatelet medication. Further study is anticipated to see whether this strategy can bring benefits regarding the clinical outcome.

Disclosures: Moon Hee Han—UNRELATED: Consultancy: Microvention.* *Money paid to the institution.

\section{REFERENCES}

1. Molyneux A, Kerr R, Stratton I, et al. International Subarachnoid Aneurysm Trial (ISAT) of neurosurgical clipping versus endovascular coiling in 2143 patients with ruptured intracranial aneurysms: a randomised trial. Lancet 2002;360:1267-74 CrossRef Medline

2. Derdeyn CP, Cross DT 3rd, Moran CJ, et al. Postprocedure ischemic events after treatment of intracranial aneurysms with Guglielmi detachable coils. J Neurosurg 2002;96:837-43 CrossRef Medline

3. Ries T, Buhk JH, Kucinski T, et al. Intravenous administration of acetylsalicylic acid during endovascular treatment of cerebral aneurysms reduces the rate of thromboembolic events. Stroke 2006;37: 1816-21 CrossRef Medline

4. Yamada NK, Cross DT 3rd, Pilgram TK, et al. Effect of antiplatelet therapy on thromboembolic complications of elective coil embolization of cerebral aneurysms. AJNR Am J Neuroradiol 2007;28: 1778-82 CrossRef Medline

5. Hwang G, Jung C, Park SQ, et al. Thromboembolic complications of elective coil embolization of unruptured aneurysms: the effect of oral antiplatelet preparation on periprocedural thromboembolic complication. Neurosurgery 2010;67:743-48 CrossRef Medline

6. Kang HS, Han MH, Kwon BJ, et al. Is clopidogrel premedication useful to reduce thromboembolic events during coil embolization for unruptured intracranial aneurysms? Neurosurgery 2010;67: 1371-76; discussion 1376 CrossRef Medline

7. Rahme RJ, Zammar SG, El Ahmadieh TY, et al. The role of antiplatelet therapy in aneurysm coiling. Neurol Res 2014;36:383-88 CrossRef Medline

8. CAPRIE Steering Committee. A randomised, blinded, trial of clopidogrel versus aspirin in patients at risk of ischaemic events (CAPRIE). Lancet 1996;348:1329-39 CrossRef Medline

9. Yusuf S, Zhao F, Mehta SR, et al. Effects of clopidogrel in addition to aspirin in patients with acute coronary syndromes without ST-segment elevation. $N$ Engl J Med 2001;345:494-502 CrossRef Medline

10. Kang HS, Kwon BJ, Kim JE, et al. Preinterventional clopidogrel response variability for coil embolization of intracranial aneurysms: clinical implications. AJNR Am J Neuroradiol 2010;31:1206-10 CrossRef Medline

11. Serebruany VL, Steinhubl SR, Berger PB, et al. Variability in platelet responsiveness to clopidogrel among 544 individuals. $\mathrm{J}$ Am Coll Cardiol 2005;45:246-51 CrossRef Medline

12. Delgado Almandoz JE, Kadkhodayan Y, Crandall BM, et al. Variability in initial response to standard clopidogrel therapy, delayed conversion to clopidogrel hyper-response, and associated thromboembolic and hemorrhagic complications in patients undergoing endovascular treatment of unruptured cerebral aneurysms. J Neurointerv Surg 2014;6:767-73 CrossRef Medline

13. Kim MS, Jo KI, Yeon JY, et al. Association between postprocedural infarction and antiplatelet drug resistance after coiling for unruptured intracranial aneurysms. AJNR Am J Neuroradiol 2016;37: 1099-105 CrossRef Medline

14. Kim B, Kim K, Jeon P, et al. Thromboembolic complications in patients with clopidogrel resistance after coil embolization for unruptured intracranial aneurysms. AJNR Am J Neuroradiol 2014;35: 1786-92 CrossRef Medline 
15. Asai T, Miyachi S, Izumi T, et al. Relationship between low response to clopidogrel and periprocedural ischemic events with coil embolization for intracranial aneurysms. J Neurointervent Surg 2016;8: 752-55 CrossRef Medline

16. Mega JL, Close SL, Wiviott SD, et al. Cytochrome P450 genetic polymorphisms and the response to prasugrel: relationship to pharmacokinetic, pharmacodynamic, and clinical outcomes. Circulation 2009;119:2553-60 CrossRef Medline

17. Gurbel PA, Bliden KP, Samara W, et al. Clopidogrel effect on platelet reactivity in patients with stent thrombosis: results of the CREST Study. J Am Coll Cardiol 2005;46:1827-32 CrossRef Medline

18. Matetzky S, Shenkman B, Guetta V, et al. Clopidogrel resistance is associated with increased risk of recurrent atherothrombotic events in patients with acute myocardial infarction. Circulation 2004;109:3171-75 CrossRef Medline

19. Hochholzer W, Trenk D, Bestehorn HP, et al. Impact of the degree of peri-interventional platelet inhibition after loading with clopidogrel on early clinical outcome of elective coronary stent placement. J Am Coll Cardiol 2006;48:1742-50 CrossRef Medline

20. Yang H, Li Y, Jiang Y, et al. Thromboelastography for monitoring platelet function in unruptured intracranial aneurysm patients undergoing stent placement. Interv Neuroradiol 2015;21:61-68 CrossRef Medline

21. Yang H, Li Y, Jiang Y. Insufficient platelet inhibition and thromboembolic complications in patients with intracranial aneurysms after stent placement. J Neurosurg 2015 Nov 20. [Epub ahead of print] Medline
22. Hwang G, Huh W, Lee JS, et al. Standard vs modified antiplatelet preparation for preventing thromboembolic events in patients with high on-treatment platelet reactivity undergoing coil embolization for an unruptured intracranial aneurysm: a randomized clinical trial. JAMA Neurol 2015;72:764-72 CrossRef Medline

23. Wiviott SD, Antman EM, Braunwald E. Prasugrel. Circulation 2010; 122:394-403 CrossRef Medline

24. Wiviott $\mathrm{SD}$, Braunwald $\mathrm{E}, \mathrm{McCabe} \mathrm{CH}$, et al. Intensive oral antiplatelet therapy for reduction of ischaemic events including stent thrombosis in patients with acute coronary syndromes treated with percutaneous coronary intervention and stenting in the TRITONTIMI 38 trial: a subanalysis of a randomised trial. Lancet 2008;371: 1353-63 CrossRef Medline

25. Wiviott SD, Braunwald E, McCabe $\mathrm{CH}$, et al. Prasugrel versus clopidogrel in patients with acute coronary syndromes. $N$ Engl J Med 2007;357:2001-15 CrossRef Medline

26. Sangkuhl K, Klein TE, Altman RB. Clopidogrel pathway. Pharmacogenet Genomics 2010;20:463-65 CrossRef Medline

27. Cattaneo M. Fast, potent, and reliable inhibition of platelet aggregation. Eur Heart J Suppl 2009;11:G9-13 CrossRef

28. Stetler WR, Chaudhary N, Thompson BG, et al. Prasugrel is effective and safe for neurointerventional procedures. J Neurointerv Surg 2013;5:332-36 CrossRef Medline

29. Akbari SH, Reynolds MR, Kadkhodayan Y, et al. Hemorrhagic complications after prasugrel (Effient) therapy for vascular neurointerventional procedures. J Neurointerv Surg 2013;5:337-43 CrossRef Medline 